\title{
Bronchial hyperresponsiveness to mannitol, airway inflammation and Asthma Control Test in atopic asthmatic children
}

\author{
Marina Attanasi ${ }^{1}$, Nicola P. Consilvio ${ }^{1}$, Daniele Rapino ${ }^{1}$, Marta Di Nicola ${ }^{2}$, Alessandra Scaparrotta ${ }^{1}$, \\ Anna Cingolani ${ }^{1}$, Marianna I. Petrosino ${ }^{1}$, Paola Di Filippo ${ }^{1}$, Sabrina Di Pillo ${ }^{1}$, Francesco Chiarelli ${ }^{3}$
}

\author{
'Department of Paediatrics, Allergy and Respiratory Diseases Clinic, \\ University of Chieti, Chieti, Italy \\ 2Laboratory of Biostatistics, Department of Experimental and Clinical Sciences, \\ University of Chieti, Chieti, Italy \\ ${ }^{3}$ Department of Paediatrics, University of Chieti, Chieti, Italy
}

Submitted: 17 December 2013

Accepted: 6 April 2014

Arch Med Sci 2016; 12, 1: 137-144

DOI: $10.5114 /$ aoms.2016.57589

Copyright @ 2016 Termedia \& Banach

\begin{abstract}
Introduction: The aim of this study was to evaluate the relationship between airway hyperresponsiveness (AHR) to mannitol and bronchial inflammation measured as exhaled nitric oxide (FeNO) and to assess whether asthma control correlates with AHR to mannitol and FeNO in atopic asthmatic children.

Material and methods: Allergy evaluation, the mannitol challenge test, FeNO levels and the Asthma Control Test (ACT) questionnaire were assessed in 40 children with intermittent and mild persistent allergic asthma.

Results: All the subjects showed positive AHR to mannitol. Pearson's correlation test revealed a significant inverse correlation between AHR (mannitol $\left.\mathrm{PD}_{15}\right)$ and FeNO $(p=0.020)$. There was also a significant positive correlation between ACT and $\mathrm{PD}_{15}(p=0.020)$ and a significant negative correlation between ACT and FeNO levels $(p=0.003)$. The study population was divided into two groups according to FeNO levels (group $A \geq 16$ ppb vs. group $B<16 p p b$ ). In group $A$ mannitol $\mathrm{PD}_{15}$ was significantly lower $(p=0.040)$ and ACT score values were significantly lower $(p=0.001)$ compared to group $B$. In group $A$, the ACT showed that $13.3 \%$ of subjects had well-controlled asthma, $80 \%$ had partially controlled asthma and $6.7 \%$ had uncontrolled asthma. In group B, the ACT showed that $72 \%$ of subjects had well-controlled asthma and $28 \%$ had partially controlled asthma.

Conclusions: Our findings indicate that the degree of AHR to mannitol correlates with the degree of airway inflammation in asthmatic atopic children; moreover, better control of asthma correlates with a lower degree of AHR to both mannitol and FeNO.
\end{abstract}

Key words: asthma, airway hyperresponsiveness, mannitol, exhaled nitric oxide, asthma control.

\section{Introduction}

Asthma is a common chronic disorder of the airway involving complex interactions among airflow obstruction, airway hyperresponsiveness (AHR), and underlying bronchial inflammation [1]. Airway inflammation and AHR are recognized as major features of bronchial asthma [2]. Because of these

\author{
Corresponding author: \\ Marina Attanasi MD \\ Allergy and Respiratory \\ Diseases Unit \\ Department of Paediatrics \\ University of Chieti \\ Chieti, Italy \\ Phone: +39 (0)871358018, \\ +393200564453 \\ Fax: +39 (0)871357590 \\ E-mail: \\ marina_attanasi@hotmail.it
}


observations, it has been suggested that monitoring of airway inflammation and bronchial responsiveness may be useful for gauging the severity of the disease and the efficacy of the anti-inflammatory treatment [3]. Moreover, airway inflammation also appears to be a major factor determining the degree of AHR [2] and may also be reflected by the levels of exhaled nitric oxide (FeNO) [4].

Airway hyperresponsiveness is usually measured by bronchial challenge with direct or indirect stimuli. Because AHR to indirect stimuli is dependent on the presence of inflammatory cells and release of their mediators in addition to a responsive muscle, it is considered more reflective of airway inflammation than airway geometry [5]. Mannitol dry powder (MDP) is a new indirect bronchial provocation test, consisting of a hyperosmolar challenge comparable to hypertonic saline (HS) solution [6]. Mannitol acts as an osmotic stimulus on the cells in the airway mucosa and is thought to simulate the dehydration of the airway surface liquid, leading to the release of bronchoconstricting mediators from inflammatory cells, and causing smooth muscle contraction in responsive individuals [7].

MDP challenge is easier, quicker to perform and better tolerated than HS or methacholine, especially in children, and it shows comparable accuracy of exercise testing in diagnosing asthma [8-10]. In the light of the above consideration, it is likely to become one of the standard bronchial challenge tests in clinical practice and the research field [11] The FeNO is a non-invasive marker of eosinophilic airway inflammation, accepted by the PRACTALL Consensus Report as a complementary item in the follow-up of bronchial inflammation [12].

Few studies have been conducted to evaluate the relationship between airway hyperresponsiveness by using the mannitol challenge test and FeNO in atopic asthmatic children [13, 14]. In fact, children with intermittent and mild persistent allergic asthma represent a special study population. The presence of atopy, as postulated by Suh DI and colleagues, could better reflect the link between AHR to mannitol and FeNO [15]. On the basis of this knowledge, the primary aim of this study was to evaluate the correlation between AHR to mannitol and bronchial inflammation measured as FeNO levels in a population of children with intermittent and mild persistent allergic asthma.

Studies in the literature have postulated that poorly or uncontrolled asthma may be associated with bronchial inflammation (in terms of increased FeNO levels). As well as airway inflammation, asthma control is likely to be influenced by the degree of AHR [16]. The recent update of the Global Initiative for Asthma (GINA) guidelines has placed emphasis on the concept of asthma control as being the key target of treatment [17]. In this direction several validated questionnaires have been proposed to assess asthma control in children and young adults $[18,19]$. Previous studies have demonstrated that the Asthma Control Test (ACT) is a useful tool in addition to clinical and functional evaluation in the management of asthma [20].

Therefore ACT was assessed in the present study for the first time, with the secondary aim to evaluate whether asthma control correlates with AHR to mannitol and FeNO.

\section{Material and methods}

\section{Study design}

This was a cross-sectional study. Subjects were asked to come for one study visit. In order to avoid any possible influence on bronchial reactivity, they were asked to refrain from taking current conventional agents in the treatment of asthma [21, 22], such as inhaled corticosteroids (ICSs), leukotriene receptor antagonists (LTRAs) and long-acting $\beta_{2}$-agonists (LABAs) for 4 weeks before the study day as a run-in period to be enrolled in the study protocol. We considered this period sufficient to wash-out from any asthma medications, as suggested by Anderson et al. [23]. During this period all children were followed closely. Every 3 days we performed phone questionnaires in order to determine any occurrence of respiratory symptoms, and parents were asked to inform us as soon as possible. On arrival, the clinical diagnosis of asthma was confirmed by a pediatric respiratory staff physician by examination and medical history. During the appointment, all children underwent FeNO analysis before the baseline lung function test measurements, as basal assessment for the mannitol challenge test protocol. Furthermore, an indirect challenge with mannitol dry powder was performed. For assessing the clinical control of asthma, before the challenge test, we asked subjects to fill out a validated Italian translation of the ACT questionnaire (ACT English version available at www.asthmacontrol.com-Italy/Italian final version 09 June 06-Mapi Research Institute).

The ACT provides numerical values to distinguish different levels of control.

\section{Subjects}

Forty asthmatic patients were recruited at the Pediatric Allergy and Respiratory Diseases Unit of the Department of Pediatrics, "G. D'Annunzio" University of Chieti, Chieti, Italy.

These 40 subjects met the American Thoracic Society-European Respiratory Society (ATS-ERS) criteria for asthma [24], had a history of wheezing and chest tightness, and were previously di- 
agnosed by a pediatric respiratory physician as having asthma. All children were classified as having intermittent or mild persistent asthma based on the GINA guidelines $[25,26]$. The skin prick test (SPTs) and total and specific serum lgE levels were performed. The study population was characterized by atopic children, who were IgE-sensitized, with a measurable level of allergen-specific IgE. Exclusion criteria for patient recruitment were: 1) history of upper and lower airway infection over the 4 weeks preceding the study; 2) congenital abnormalities of cardio-respiratory system, chest or skeletal deformities, or neuromuscular system disease, chronic and autoimmune disease, gastro-esophageal reflux; 3) any other condition impeding performance of lung function tests.

Written informed consent was obtained from all parents and children older than 12 years, and oral consent from all children.

The study was approved by the Ethical Committee of the University of Chieti.

\section{Mannitol challenge test}

The mannitol challenge test (MCT) was performed according to the protocol of Anderson and co-workers using a single dose Inhalator (Boehringer Ingelheim, Ingelheim, Germany) [7]. The dose protocol consisted of 0 (empty capsule acting as a placebo), 5, 10, 20, 40, 80, 160, 160 and $160 \mathrm{mg}$ mannitol, resulting in a maximum cumulative dose of $635 \mathrm{mg}$. The 80-mg and 160-mg doses were given in multiples of $40 \mathrm{mg}$ capsules. Children were asked to inhale from the device from near to functional residual capacity (FRC) to near to total lung capacity (TLC), and to subsequently hold their breath for $5 \mathrm{~s}$. Children had a nose clip on during inhalation and were asked to exhale through their mouth to minimize deposition in the nasopharynx. Three forced expiratory maneuvers were performed $60 \mathrm{~s}$ after each dose, and the highest forced expiratory volume in $1 \mathrm{~s}\left(\mathrm{FEV}_{1}\right)$ measurement was recorded. The challenge was stopped when a $15 \%$ decrease in $\mathrm{FEV}_{1}$ was measured or a total cumulative dose of $635 \mathrm{mg}$ had been administered. The $\mathrm{FEV}_{1}$ value measured after the $0 \mathrm{mg}$ capsule is taken as the baseline $\mathrm{FEV}_{1}$ and is used to calculate the percentage decrease in $\mathrm{FEV}_{1}$ in response to the mannitol challenge. The provoking dose of mannitol to induce a $15 \%$ fall in $\mathrm{FEV}_{1}$ for mannitol was calculated $\left(\mathrm{PD}_{15}\right)$.

\section{Atopic sensitization}

Atopy was documented by elevated specific serum IgE or by a positive SPTs to at least one of the aero-allergens [27]. SPTs were performed following the European Academy of Allergy and Clinical Immunology (EAACI) guidelines. A positive result was defined as a wheal at least $3 \mathrm{~mm}$ in diameter in response to one or more allergens [28].

Determination of allergen-specific lgE, expressed as kUA/l, was made by an Immunoenzymatic Allergo-sorbent Test (Cap test Pharmacia) [29]. The confidence interval is from 0.35 (class 1 ) to $100 \mathrm{kUA} / \mathrm{l}$ (class 5). Class 0 is the default for values up to $0.35 \mathrm{kUA} / \mathrm{l}$, and class 6 is the default for values over $100 \mathrm{kUA} / \mathrm{l}$. The range of positive values of specific serum IgE was above $0.35 \mathrm{kUA} / \mathrm{l}$.

\section{Inflammatory markers}

In order to evaluate bronchial inflammation status, all children underwent FeNO analysis.

FeNO was determined with an on-line method using a single breath exhalation and a sensitive chemiluminescence assay (Ecomedics CLD 88), according to ATS-ERS [30]. Patients made an inspiration of eNO-free air via a mouthpiece immediately followed by full exhalation at a constant rate $(50 \mathrm{ml} / \mathrm{s})$ for at least $5 \mathrm{~s}$. The mean of three acceptable readings at the end of the expiration (plateau phase) was taken as the representative value for each measurement, according to ATS-ERS criteria [31]. The cut-off point for an increased level of FeNO was defined as $16 \mathrm{ppb}$ according to the literature, which showed that FeNO levels higher than $16 \mathrm{ppb}$ had the highest diagnostic value to confirm exercise-induced bronchospasm [32].

\section{Asthma Control: ACT (Asthma Control Test)}

The ACT is a straightforward, self-administered questionnaire with five questions on asthma symptoms (such as shortness of breath, wheezing, coughing); use of rescue medications (such as albuterol or salbutamol); and effect of asthma on daily functioning (such as waking up at night or earlier than usual in the morning). For children younger than 11 years the C-ACT questionnaire was used [19], while for children older than 12 years the ACT questionnaire developed by $\mathrm{Na}$ than et al. was used [18]. Each item included five response options for children and parents, from 1 (worst) to 5 (best). The lowest and highest possible scores were thus 5 (totally uncontrolled asthma) and 25 (total asthma control), respectively, and a score of 19 or less was shown to be indicative of poorly controlled asthma $[18,19]$.

\section{Statistical analysis}

The primary outcome of this study was the correlation between FeNO levels (expressed in ppb) and AHR to mannitol in terms of $\mathrm{PD}_{15}$. According to data from the literature and clinical experience of the involved investigators, we expected a correlation coefficient of 0.45 between the above 
two quantitative variables evaluated in this study. Assuming a type I error $(\alpha)$ of 0.05 , a group of 35 patients would provide a power $(1-\beta)$ of $80 \%$ to detect a difference of 0.45 in the correlation coefficient. These calculations were performed using PASS 2005 (Kaysville, Utah).

All quantitative parameters were expressed as mean \pm standard deviation (SD), and all categorical variables were reported as frequency and percentage.

Best values of spirometric measurements of $\mathrm{FEV}_{1}$ were considered for statistical evaluation.

The provocative dose of mannitol causing a fall in $\mathrm{FEV}_{1}$ of $15 \%$ was calculated for all children $\left(\mathrm{PD}_{15}\right.$; measuring airway sensitivity).

The relationship between mannitol $\mathrm{PD}_{15}$ and FeNO was reported graphically as a scattergram and evaluated by Pearson's correlation coefficient (rp).

After dividing our study population into two groups on the basis of FeNO levels (group $A \geq 16 \mathrm{ppb}$ and group $B<16$ ppb - cut-off with the highest diagnostic value for exercise-induced bronchoconstriction (EIB)) [32], Student's unpaired $t$ test was applied to compare the two groups for quantitative parameters. The $\chi^{2}$ test was performed to evaluate the difference between groups for categorical variables, and Fisher's exact test was used to evaluate the prevalence of allergen sensitization.

A $p$-value less than 0.05 was considered to be statistically significant. All calculations were made with the computer program Statistical Package for the Social Sciences (SPSS), version 16.0 software for Windows.

\section{Results}

Forty children (24 males and 16 females) with a mean age of $9.3 \pm 2.9$ years with intermittent and mild persistent asthma were enrolled. Population characteristics are shown in Table I.

All children underwent a complete clinical and medical history questionnaire evaluation, including questions about parasitic diseases or other allergic diseases, and upper or lower respiratory illnesses. None of the enrolled children suffered from these diseases.

During the enrollment 2 children were excluded because they were unable to perform the MCT as their $\mathrm{FEV}_{1}$ values were under $70 \%$ of the predicted value for age, gender and height, and one because he was not able to perform reproducible spirometry.

All children had a positive response to MCT, and were IgE-sensitized with a measurable level of allergen-specific IgE ( $\geq 0.35 \mathrm{kUA} / \mathrm{l})$; thus they were considered atopic.

Pearson's correlation test revealed a significant inverse correlation between airway respon- siveness (mannitol $\mathrm{PD}_{15}$ ) and FeNO ( $r p=-0.36$; $p=0.020$ ), as shown in Figure 1 . There was also a significant positive correlation between ACT and $\mathrm{PD}_{15}(r p=0.36 ; p=0.020)$ and a significant negative correlation between ACT and FeNO levels $(r p=-0.4 ; p=0.003)$.

Based on the results of FeNO values, the study population was divided into two groups: group $A$ $(n=15)$ with FeNO values equal to or above the cut-off of $16 \mathrm{ppb}$; group B $(n=25)$ with FeNO values under the cut-off of $16 \mathrm{ppb}$.

In group $A$, mean mannitol $\mathrm{PD}_{15}$ was 154.7 $\pm 97.5 \mathrm{mg}$, and mean FeNO was $42.8 \pm 20.1 \mathrm{ppb}$, while in group B, mean PD15 was $262.8 \pm 186.5$ $\mathrm{mg}$, and mean FeNO was $7.17 \pm 4.3 \mathrm{ppb}$.

Regarding the assessment of clinical control of asthma, in group A ACT showed that $13.3 \%$ (2 of $15)$ of subjects of this group had controlled asthma (ACT score 25), $80 \%$ (12 of 15) of subjects had partly controlled asthma (ACT score 20-24) and $6.7 \%(1 / 15)$ had uncontrolled asthma (ACT < 19). In group B ACT showed that $72 \%$ (18 of 25 ) of subjects of this group had well-controlled asthma (ACT score 25), and 28\% (7 of 25) of subjects had partly controlled asthma (ACT score 20-24) and nobody had uncontrolled asthma.

Finally, using Student's unpaired $t$ test, we found that group A had significantly lower values of mannitol PD $_{15}$ compared to group $B$ (154.7 $\pm 97.5 \mathrm{mg}$ vs. $262.8 \pm 186.5 \mathrm{mg} ; p=0.040$ ), and significantly lower values of ACT score (22.1 vs. 24.4; $p=0.001$ ) (Table I).

\section{Discussion}

The results of the present study revealed that the AHR to mannitol correlates with FeNO levels and ACT score.

In the literature, there are few studies that evaluate the correlation between the MCT and bronchial inflammation by FeNO in childhood [11, $13,14]$. In addition, no study correlates this challenge test with a validated asthma questionnaire.

Several studies have suggested a causal relationship between airway inflammation and hyperresponsiveness in allergic asthma based mainly on the observation that acute exposure to allergens caused enhanced airway responsiveness and inflammatory cell recruitment in the airways [33, 34]. In the study of Leuppi et al. [35], raised FeNO levels, in atopic children, are associated with AHR, suggesting that exhaled NO is more than just a marker for atopy. Moreover, our data appear to be consistent with the findings of Sverrild et al. [13], who found in young adults a close association between AHR to mannitol and ongoing airway inflammation.

From the results of this study it also appeared that better control of asthma was linked with 
Table I. Characteristics of the study population

\begin{tabular}{|c|c|c|c|}
\hline Parameter & Group A $(n=15)$ & Group B $(n=25)$ & Value of $p^{a}$ \\
\hline Age [years] & $10.3 \pm 2.3$ & $8.7 \pm 2.6$ & 0.099 \\
\hline Weight [kg] & $42.7 \pm 14.3$ & $35.6 \pm 11.5$ & 0.089 \\
\hline Height $[\mathrm{cm}]$ & $144.9 \pm 18.9$ & $134.5 \pm 14.3$ & 0.060 \\
\hline Gender (M/F) & $8 / 7$ & $16 / 9$ & $0.739^{b}$ \\
\hline Total serum IgE $\left[\mathrm{kU}_{\mathrm{A}} / \mathrm{l}\right]$ & $350.3 \pm 331.8$ & $321.4 \pm 345.6$ & 0.698 \\
\hline FeNO [ppb] & $42.8 \pm 20.1$ & $7.2 \pm 4.3$ & $<0.001$ \\
\hline $\mathrm{FEV}_{1}$ baseline (\%-predicted) & $103.4 \pm 10.5$ & $111.7 \pm 16.5$ & 0.096 \\
\hline \multicolumn{4}{|l|}{ Classification of asthma: } \\
\hline Intermittent vs. mild persistent & $10 / 5$ & $22 / 3$ & \\
\hline \multicolumn{4}{|l|}{ Treatment: } \\
\hline On-therapy before the study: & 5 & 3 & \\
\hline ICS [ug/day] & 100 & 100 & \\
\hline LABA [mg/day] & - & - & - \\
\hline ICS + LABA [ $\mu \mathrm{g} / \mathrm{day}+\mathrm{mg} / \mathrm{day}]$ & - & - & - \\
\hline Antihistamines [mg/day] & - & - & - \\
\hline Off-therapy before the study & 10 & 22 & \\
\hline \multicolumn{4}{|l|}{ Prevalence of allergen sensitization: } \\
\hline Dermatophagoides pt. and fa. (\%) & 86.7 & 92.1 & $0.624^{c}$ \\
\hline Rye grass (\%) & 60.1 & 56.2 & $0.990^{c}$ \\
\hline Olive tree (\%) & 46.7 & 32.2 & $0.502^{c}$ \\
\hline Wall pellitory (\%) & 46.7 & 20.1 & $0.091^{c}$ \\
\hline Cat epithelia (\%) & 26.7 & 20.2 & $0.705^{c}$ \\
\hline Alternaria alternate (\%) & 6.7 & 12.1 & $0.990^{c}$ \\
\hline Aspergillus spp. (\%) & 6.7 & 0.9 & $0.375^{c}$ \\
\hline \multicolumn{4}{|c|}{ Bronchial hyperresponsiveness and asthma control: } \\
\hline AHR to mannitol $\left(\mathrm{PD}_{15}\right)[\mathrm{mg}]$ & $154.7 \pm 97.5$ & $262.8 \pm 186.5$ & 0.040 \\
\hline ACT score & $22.1 \pm 2.4$ & $24.4 \pm 1.1$ & 0.001 \\
\hline
\end{tabular}

Values are mean $\pm S D$ or number. ${ }^{a}$ Student's unpaired $t$ test, ${ }^{\mathrm{b}}$ Chi-squared test, ${ }^{\mathrm{c}}$ Fisher's Exact test, $M-$ male, $F-$ female, $_{\text {FEV }}-$ forced $_{1}$ expiratory volume in $1 \mathrm{~S}, P D_{15}$ - provocative dose causing a $15 \%$ decline in FEV ${ }_{1}$, FeNO -fractional exhaled nitric oxide, AHR - airway hyperresponsiveness, $A C T$ - asthma control test.

a lower degree of AHR to mannitol and improved control of bronchial inflammation. In keeping with these findings, there are studies that have shown that ACT score reflects lung function and bronchial inflammation $[36,37]$ and have confirmed that $\mathrm{ACT}$ is complementary to other markers of disease control in asthmatic children, especially in the context of follow-up visits [38].

However, the relationship between the asthma control questionnaire and AHR remains unclear. Many studies have shown that the ACT has no relationship with airway hyperresponsiveness eval- uated by the Bruce Protocol and other stress tests $[39,40]$. In contrast with these observations, our results for the MCT showed that ACT had a direct correlation with AHR and airway inflammation. This apparent contradiction could be explained by the fact that the ACT questionnaire does not include questions about exercise-induced symptoms. As a matter of fact, the ACT and exercise-induced bronchoconstriction measure separate domains of asthma control. Another explanatory factor could be that "poor-perceiver" asthmatic children might modify their behavior and avoid physical activity 


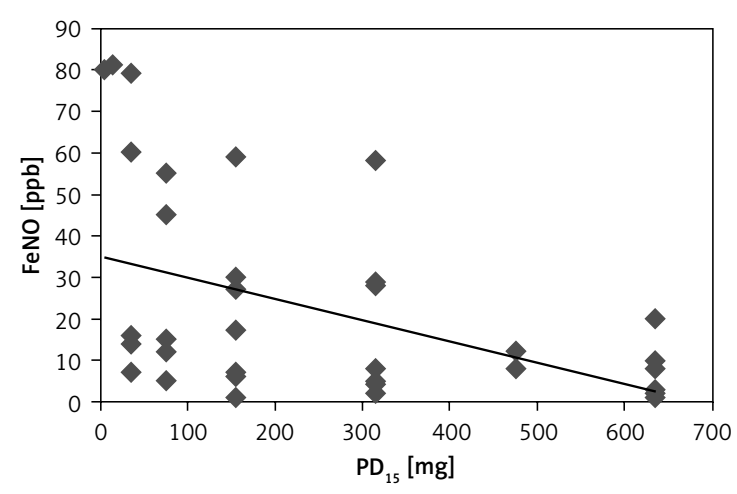

Figure 1. Scattergram between FeNO and PD15

FeNO - exhaled nitric oxide, PD15 - provocative dose causing a fall in FEV of $15 \%$.

that could be a trigger for bronchospasm, leading to a vicious circle.

This suggests that AHR to mannitol has a close correlation not only with inflammation but also with clinical symptoms related to asthma. This challenge test is probably more sensitive than the other indirect stimuli in atopic asthmatic children in the detection of AHR and asthma control.

The mannitol challenge test might be used in children with poorly perceived asthma symptoms, with only apparently well-controlled asthma, or in children with EIB in which FeNO levels are elevated [13]. As a matter of fact, a positive test predicts active asthma and potential for EIB, while a negative test might suggest good control of asthma [41]. Moreover, response-dose ratio may be used to monitor an intervention or back titration of steroid dose [42].

The relationship between FeNO and AHR in atopic children indicated in our research is confirmed by several existing studies. In agreement with this suggestion, Steerenberg et al. [43] demonstrate that the presence of AHR was positively associated with FeNO only in atopic children. Lúdvíksdóttir et al. [44] reported a similar relationship in asthmatic adults. There is a well-established relationship between atopy and increased airway responsiveness in children $[45,46]$; in fact our data suggest that increased FeNO values may be associated with a mechanism linking these two factors. This may involve inflammatory processes and would support the hypothesis that FeNO is a marker of allergic airway inflammation [47].

Contrary to our findings, many studies have shown a dissociation between airway inflammation and AHR in children with mild intermittent/ mild persistent asthma. Particularly, Silvestri et al. [48] indicated that FeNO levels did not seem to be accurate predictors of the degree of AHR, measured by using a methacholine challenge test, in children with intermittend/mild persistent asthma. Although the authors reported that the study was of insufficient statistical power, in our opinion a possible explanation for this could be that the response to mannitol is mediated mainly through mast cells, which are responsible for the main release of bronchoconstricting mediators $[49,50]$.

Some limitations of the present study need to be mentioned, such as small sample size and the inclusion of only allergic subjects. Another limitation is the cross-sectional design that limits the possibility to prove the causal relationship. Therefore, additional clinical studies on larger populations including non allergic asthmatic children, with a longitudinal design, are necessary to validate the preliminary data of this study. Our hypothesis is that FeNO reflects eosinophilic inflammation, commonly present in children with allergic asthma. On the other hand, mannitol stimulus recruits many inflammatory cells, not only eosinophils, but also mast cells and their mediators, and actually we do not know which is the main pathway that leads to smooth muscle bronchoconstriction. Further studies on the pathophysiological mechanism of mannitol are needed, and particularly in this kind of asthmatic population.

Another shortcoming of the study is the variability of mannitol- $\mathrm{PD}_{15}$, which in our view depends on the small sample study population, not influenced by the treatment. Again the presence of one child in group A with an ACT score value $<19$ may have skewed our results. Nevertheless, we suppose that this value really did not influence our results. As a matter of fact, this patient presented some mild asthma exacerbations 2 weeks before the lung function evaluation and symptoms during exercise that required only "rescue medications" (short-acting $\beta_{2}$-agonists), for which a period of $8 \mathrm{~h}$ was considered sufficient to wash-out [23].

The MCT is safe, available as a convenient and standardized test kit with prefilled dry powder capsules and a single use dry powder inhaler device, employs a standard operating procedure for dose and delivery, and is easier especially in children, with only some cough during the challenge [51].

In conclusion, the results of this study indicate that the mannitol challenge test could be a useful diagnostic tool in clinical practice in order to show a better clinical correlation with asthma symptoms in intermittent and mild persistent atopic asthmatic children.

\section{Acknowledgments}

We thank all the nurses of the Department of Pediatrics, University of Chieti, in particular Di Luca Dentina and Diomedi Angela, for their daily support in clinical research and care of children.

\section{Conflict of interest}

The authors declare no conflict of interest. 


\section{References}

1. Piotrowski WJ, Majewski S, Marczak J, Kurmanowska Z, Górski P, Antczak A. Exhaled breath 8-isoprostane as a marker of asthma severity. Arch Med Sci 2012; 8: 515-20.

2. National Asthma Education and Prevention Program. Expert panel report 3: Guidelines for the diagnosis and management of asthma-Summary Report 2007. J Allergy Clin Immunol 2007; 120 (Suppl. 5): S94-138.

3. Hastie AT, Moore WC, Meyers DA, et al. Analyses of asthma severity phenotypes and inflammatory proteins in subjects stratified by sputum granulocytes. J Allergy Clin Immunol 2010; 125: 1028-36.

4. Thomas PS, Gibson PG, Wang H, Shah S, Henry RL. The relationship of exhaled nitric oxide to airway inflammation and responsiveness in children. J Asthma 2005; 42: 291-5.

5. Cockcroft DW, Davis BE. Mechanisms of airway hyperresponsiveness. J Allergy Clin Immunol 2006; 118: 551-9.

6. Brannan JD, Anderson SD, Perry CP, Freed-Martens R, Lassig AR, Charlton B; Aridol Study Group. The safety and efficacy of inhaled dry powder mannitol as a bronchial provocation test for airway hyperresponsiveness: a phase 3 comparison study with hypertonic (4.5\%) saline. Respir Res 2005; 6: 144.

7. Anderson SD, Brannan J, Spring J, et al. A new method for bronchial-provocation testing in asthmatic subjects using a dry powder of mannitol. Am J Respir Crit Care Med 1997; 156: 758-65.

8. Barben J, Roberts M, Chew N, Carlin JB, Robertson CF. Repeatability of bronchial responsiveness to mannitol dry powder in children with asthma. Pediatr Pulmonol 2003; 36: 490-4.

9. Barben J, Kuehni CE, Strippoli MP, Schiller B, Hammer J, Trachsel D; Swiss Paediatric Respiratory Research Group. Mannitol dry powder challenge in comparison with exercise testing in children. Pediatr Pulmono 2011; 46: 842-8.

10. Kersten ET, Driessen JM, van der Berg JD, Thio BJ. Mannitol and exercise challenge tests in asthmatic children. Pediatr Pulmonol 2009; 44: 655-61.

11. Decimo F, Capristo C, Amelio R, Maiello N, Capristo AF, Miraglia Del Giudice M. Evaluation of bronchial hyperreactivity with mannitol dry powder challenge test in a paediatric population with intermittent allergic asthma or allergic rhinitis. Int J Immunopathol Pharmacol 2011; 24: 1069-74.

12. Bacharier LB, Boner A, Carlsen KH, et al. European Pediatric Asthma Group. Diagnosis and treatment of asthma in childhood: a PRACTALL Consensus Report. Allergy 2008; 63: 5-34.

13. Sverrild A, Porsbjerg C, Thomsen SF, Backer V. Airway hyperresponsiveness to mannitol and methacoline and exhaled nitric oxide: a random-sample population study. J Allergy Clin Immunol 2010; 126: 952-8.

14. Barben J, Strippoli MF, Trachsel D, Schiller B, Hammer J, Kuehni CE. Effect of mannitol dry powder challenge on exhaled nitric oxide in children. PLoS One 2013; 8: e54521.

15. Suh DI, Lee JK, Kim CK, Koh YY. Methacholine and adenosine 5'-monophosphate (AMP) responsiveness, and the presence and degree of atopy in children with asthma. Pediatr Allergy Immunol 2011; 22: e101-6.

16. Sippel JM, Holden WE, Tilles SA, et al. Exhaled nitric oxide levels correlate with measures of disease control in asthma. J Allergy Clin Immunol 2000; 106: 645-50.
17. Koshak EA. Classification of asthma according to revised 2006 GINA: evolution from severity to control. Ann Thorac Med 2007; 2: 45-6.

18. Nathan RA, Sorkness CA, Kosinski M, et al. Development of the asthma control test: a survey for assessing asthma control. J Allergy Clin Immunol 2004; 113: 59-65.

19. Liu AH, Zeiger R, Sorkness C, et al. Development and cross-sectional validation of the Childhood Asthma Control Test. J Allergy Clin Immunol 2007; 119: 817-25.

20. Ko FW, Hui DS, Leung TF, Chu HY, Wong GW, Tung AH. Evaluation of the asthma control test: a reliable determinant of disease stability and a predictor of future exacerbations. Respirology 2012; 17: 370-8.

21. Marandi Y, Farahi N, Hashjin GS. Asthma: beyond corticosteroid treatment. Arch Med Sci 2013; 9: 521-6.

22. Mathew J, Aronow WS, Chandy D. Therapeutic options for severe asthma. Arch Med Sci 2012; 8: 589-97.

23. Anderson SD, Charlton B, Weiler JM, Nichols S, Spector SL, Pearlman DS; A305 Study Group. Comparison of mannitol and methacholine to predict exercise-induced bronchoconstriction and a clinical diagnosis of asthma. Respir Res 2009; 10: 4

24. Laszlo G. Standardization of lung function testing: helpful guidance from the ATS/ERS Task Force. Thorax 2006; 61: 744-6.

25. Global Strategy for Asthma Management and Prevention, Global Initiative for Asthma (GINA) World Report 2006. Available from: http//www.ginasthma.org.

26. National Asthma Education and Prevention Program. NAEPP expert panel report guidelines for the diagnosis and management of asthma-update on selected topics 2002. J Allergy Clin Immunol 2002; 110 (Suppl. 5): S141-219.

27. Johansson SG, Hourihane JO, Bousquet J, et al. A revised nomenclature for allergy. An EAACl position statement from the EEACI nomenclature task force. Allergy 2001; 56: 813-24.

28. Skin tests used in type I allergy testing Position paper. Sub-Committee on Skin Tests of the European Academy of Allergology and Clinical Immunology. Allergy 1989; 44 (Suppl. 10): S1-59.

29. Wolkerstorfer A, Wahn U, Kjellman NI, Diepgen TL, De Longueville M, Oranje AP. Natural course of sensitization to cow's milk and hen's egg in childhood atopic dermatitis: ETAC Study Group. Clin Exp Allergy 2002; 32: 70-3.

30. American Thoracic Society, European Respiratory Society. ATS/ERS recommendations for standardized procedures for the online and offline measurement of exhaled lower respiratory nitric oxide and nasal nitric oxide, 2005. Am J Respir Crit Care Med 2005; 171: 912-30.

31. Baraldi E, de Jongste JC. Measurement of exhaled nitric oxide in children. Eur Respir J 2002; 20: 223-37.

32. Grzelewski T, Grzelewska A, Majak P, et al. Fractional exhaled nitric oxide ( $\mathrm{FeNO}$ ) may predict exercise-induced bronchoconstriction (EIB) in schoolchildren with atopic asthma. Nitric Oxide 2012; 27: 82-7.

33. Rossi GA, Crimi E, Lantero $S$, et al. The late-phase asthmatic reaction to inhaled allergen is associated with an early recruitment of eosinophils in the airways. Am Rev Respir Dis 1991; 144: 379-83.

34. Kharitonov SA, O'Connor BJ, Evans DJ, Barnes PJ. Allergen induced late asthmatic reactions are associated with elevation of exhaled nitric oxide. Am J Respir Crit Care Med 1995; 151: 1894-9.

35. Leuppi JD, Downs SH, Downie SR, Marks GB, Salome CM. Exhaled nitric oxide levels in atopic children: relation 
to specific allergic sensitization, AHR, and respiratory symptoms. Thorax 2002; 57: 518-23.

36. Alvarez-Gutiérrez FJ, Medina-Gallardo JF, Pérez-Navarro P, et al. Comparison of the Asthma Control Test (ACT) with lung function, levels of exhaled nitric oxide and control according to the Global Initiative for Asthma (GINA). Arch Bronconeumol 2010; 46: 370-7.

37. Papakosta D, Latsios D, Manika K, Porpodis K, Kontakioti E, Gioulekas D. Asthma control test is correlated to FEV1 and nitric oxide in Greek asthmatic patients: influence of treatment. J Asthma 2011; 48: 901-6.

38. Piacentini GL, Peroni DG, Bodini A, et al. Childhood Asthma Control Test and airway inflammation evaluation in asthmatic children. Allergy 2009; 64: 1753-7.

39. Rapino D, Consilvio NP, Scaparrotta A, et al. Relationship between exercise-induced bronchospasm (EIB) and asthma control test (ACT) in asthmatic children. J Asthma 2011; 48: 1081-4.

40. Chinellato I, Piazza M, Sandri M, et al. Evaluation of association between exercise-induced bronchoconstriction and childhood asthma control test questionnaire scores in children. Pediatr Pulmonol 2012; 47: 226-32.

41. Porsbjerg C, Sverrild A, Backer V. The usefulness of the mannitol challenge test for asthma. Expert Rev Respir Med 2013; 7: 655-63.

42. Sverrild A, Porsbjerg C, Thomsen SF, Backer V. Diagnostic properties of inhaled mannitol in the diagnosis of asthma: a population study. J Allergy Clin Immunol 2009; 124: 928-32.

43. Steerenberg PA, Janssen NAH, de Meer G, et al. Relationship between exhaled NO, respiratory symptoms, lung function, bronchial hyperresponsiveness, and blood eosinophilia in school children. Thorax 2003; 58: 242-5.

44. Lúdvíksdóttir D, Janson C, Högman $M$, Hedenström $H$, Björnsson E, Boman G. Exhaled nitric oxide and its relationship to airway responsiveness and atopy in asthma. BHR-Study Group. Respir Med 1999; 93: 552-6.

45. Sears MR, Burrows B, Herbison GP, Flannery EM, Holdaway MD. Atopy in childhood. III. Relationship with pulmonary function and airway responsiveness. Clin Exp Allergy 1993; 23: 957-63.

46. Peat JK, Salome CM, Woolcock AJ. Longitudinal changes in atopy during a 4-year period: relation to bronchial hyperresponsiveness and respiratory symptoms in a population sample of Australian schoolchildren. J Allergy Clin Immunol 1990; 85: 65-74.

47. Thomas PS, Gibson PG, Wang H, Shah S, Henry RL. The relationship of exhaled nitric oxide to airway inflammation and responsiveness in children. J Asthma 2005; 42: 291-5.

48. Silvestri M, Spallarossa D, Battistini E, Brusasco V, Rossi GA. Dissociation between exhaled nitric oxide and hyperresponsiveness in children with mild intermittent asthma. Thorax 2000; 55: 484-8.

49. Porsbjerg C, Lund TK, Pedersen L, Backer V. Inflammatory subtypes in asthma are related to airway hyperresponsiveness to mannitol and exhaled NO. J Asthma 2009; 46: 606-12.

50. Brannan JD, Gulliksson M, Anderson SD, Chew N, Kumlin N. Evidence of mast cell activation and leukotriene release after mannitol inhalation. Eur Respir J 2003; 22: 491-6.

51. Sverrild A, Porsbjerg C, Backer V. Diagnostic properties of inhaled mannitol in the diagnosis of asthma: a population study. J Allergy Clin Immunol 2009; 124: 928-32. 